\title{
DIFFERENTIABILITY THEOREMS FOR WEAK SOLUTIONS OF NONLINEAR ELLIPTIC DIFFERENTIAL EQUATIONS ${ }^{1}$
}

BY CHARLES B. MORREY, JR.

I shall begin by speaking about the extremals of an integral of the form

$$
I(z, G)=\int_{G} f[x, z(x), \nabla z(x)] d x
$$

where $G$ is a domain in $R^{p}$,

$$
x=\left(x^{1}, \cdots, x^{\nu}\right), \quad z=\left(z^{1}, \cdots, z^{N}\right), \quad d x=d x^{1} \cdots d x^{\nu},
$$

$z(x)$ is a vector function, $\nabla z$ denotes its gradient which is the set of functions $\left\{z_{, \alpha}^{i}\right\}$ where $z_{, \alpha}^{i}$ denotes $\partial z^{i} / \partial x^{\alpha}$, and $f(x, z, p)\left(p=\left\{p_{\alpha}^{i}\right\}\right)$ is generally assumed continuous in all its arguments. The integrals $\int_{a}^{b}\left(1+(d z / d x)^{2}\right)^{1 / 2} d x$ and $\iint_{G}\left[\left(\partial z / \partial x^{1}\right)^{2}+\left(\partial z / \partial x^{2}\right)^{2}\right] d x^{1} d x^{2}$ are familiar examples of integrals of the form (1) in which $N=1$ in both cases, $\nu=1$ in the first case, and $\nu=2$ in the second case and the corresponding functions $f$ are defined, respectively, by

$$
f(x, z, p)=\left(1+p^{2}\right)^{1 / 2}, \quad f(x, z, p)=\left(p_{1}\right)^{2}+\left(p_{2}\right)^{2}
$$

where we have omitted the superscripts on $z$ and $p$ since $N=1$. The second integral is a special case of the Dirichlet integral which is defined in general by

$$
D(z, G)=\int_{G}|\nabla z|^{2} d x, \quad f(x, z, p)=|p|^{2}=\sum_{i, \alpha}\left(p_{\alpha}^{i}\right)^{2} .
$$

Another example is the area integral

$$
A(z, G)=\iint_{G}\left(\left[\frac{\partial\left(z^{2}, z^{3}\right)}{\partial\left(x^{1}, x^{2}\right)}\right]^{2}+\left[\frac{\partial\left(z^{3}, z^{1}\right)}{\partial\left(x^{1}, x^{2}\right)}\right]^{2}\right.
$$

$$
\left.+\left[\frac{\partial\left(z^{1}, z^{2}\right)}{\partial\left(x^{1}, x^{2}\right)}\right]^{2}\right)^{1 / 2} d x^{1} d x^{2}
$$

which gives the area of the surface

$$
z^{i}=z^{i}\left(x^{1}, x^{2}\right), \quad\left(x^{1}, x^{2}\right) \in G, \quad i=1,2,3 .
$$

1 Presidential address delivered before the Annual Meeting of the Society in New Orleans on January 24, 1969; received by the editors February 24, 1969. 
It is to be noticed that the area integral has the special property that it is invariant under diffeomorphisms (1-1 differentiable mappings, etc.) of the domain $G$ into other domains. This is the first example of an integral in parametric form.

If $f$ is of class $C^{n}$ in the arguments $(z, p)$, then $I$ is a functional of class $C^{n}$ defined on the Banach space $C^{1}(\bar{G})$. For each $z$ and $\zeta$, we define the first variation (if $n \geqq 1) I_{1}(z, \zeta, G)$ by

$$
I_{1}(z, \zeta ; G)=\phi^{\prime}(0) \text { where } \phi(\lambda)=I(z+\lambda \zeta) .
$$

This is also the Fréchet differential of $I$. In case $z \in C^{1}(\bar{G})$ and furnishes a relative minimum or maximum among such functions with the same boundary values, then

$$
I_{1}(z, \zeta ; G)=0 \quad \forall \zeta \in C^{1}(\bar{G}) \ni \zeta=0 \quad \text { on } \partial G .
$$

Let us first consider the case where $N=\nu=1$. Then (7) becomes

$$
\begin{aligned}
\int_{a}^{b}\left\{f_{p}\left[x, z(x), z^{\prime}(x)\right] \cdot \zeta^{\prime}(x)+f_{z}\left[x, z(x), z^{\prime}(x)\right] \cdot \zeta(x)\right\} d x & =0 \\
(G & =(a, b)) .
\end{aligned}
$$

If we now assume that $f$ and $z \in C^{2}(\bar{G})$, then we may integrate the first term of (8) by parts and obtain

$$
\begin{gathered}
\int_{a}^{b} \zeta(x)\left[B(x)-A^{\prime}(x)\right] d x=0, \quad A(x)=f_{p}\left[x, z(x), z^{\prime}(x)\right], \\
B(x)=f_{z}[x, z(x), p(x)]
\end{gathered}
$$

since $\zeta(a)=\zeta(b)=0$. Thus we find that $z$ satisfies Euler's equation

$$
d f_{p} / d x=f_{z} \text { or } f_{p p} z^{\prime \prime}+f_{p z} z^{\prime}+f_{p x}=f_{z} \text {. }
$$

We notice that this equation is nonlinear (in general) and of the second order. It is, however, linear in $z^{\prime \prime}$; such an equation is called quasi-linear. The equation evidently becomes singular if $f_{p p}=0$. Hence regular variational problems are those for which $f_{p p}$ never vanishes; in that case, it is assumed that $f_{p p}>0$ (so that $f$ is convex in $p$ ) and this makes minimum problems more natural than maximum problems.

We notice that if $z$ is of class $C^{2}$, then the equations (10) and (8) (assuming $\zeta=0$ on $\partial G$ ) are equivalent. However, equation (8) makes sense even if $z$ is merely of class $C^{1}$. In case $z$ is known to be at least of class $C^{1}$ and to satisfy (8), we say that $z$ is a weak solution of equation (10). It satisfies (10) in some distribution sense. 
In the general case, if $z\left(=\left(z^{1}, \cdots, z^{N}\right)\right)$ is of class $C^{1}(\bar{G})$ and furnishes a relative minimum to $I$ among all vectors $\in C^{1}(\bar{G})$ which have the same boundary values on $\partial G$, then (7) holds. If we assume that $f \in C^{2}, z \in C^{2}(\bar{G})$ and $G \in C^{1}$, the technique of (8)-(10) shows that the vector $z$ satisfies the system of Euler equations

$$
\begin{aligned}
& \frac{\partial}{\partial x^{\alpha}} f_{p_{\alpha}^{i}}=f_{z^{i}} \text { or } f_{p_{\alpha}^{i} p_{\beta}^{j} z, \alpha \beta}^{j}+f_{p_{\alpha^{j}}^{i} j z_{, \alpha}^{j}}^{j}+f_{p_{\alpha}^{i} x^{\alpha}}=f_{z^{i}} \\
& i=1, \cdots, N \text {; }
\end{aligned}
$$

a quasi-linear system. But if we merely assume $z \in C^{1}(\bar{G})$ and satisfies (7), we say that $z$ is a weak solution of the equations (11).

Now if we define $\phi(\lambda)$ as in (6), we see that $\phi^{\prime \prime}(0) \geqq 0$, where $\phi^{\prime \prime}(0)$ is called the second variation and is given by

$$
\begin{aligned}
& \phi^{\prime \prime}(0) \equiv I_{2}(z, \zeta ; G)
\end{aligned}
$$

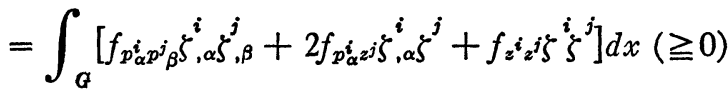

$$
\begin{aligned}
& (\zeta=0 \text { on } \partial G) \text {. }
\end{aligned}
$$

From (12) it follows (see [38, pp. 10, 11]) that

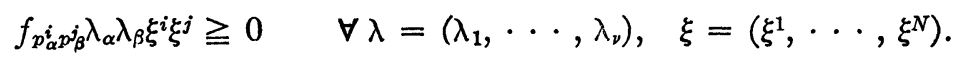

Regular problems are those for which the form (13) is positive for all $\lambda \neq 0$ and $\xi \neq 0$. If $N=1$ or $\nu=1$, the condition (13) implies the convexity of $f$ as a function of $p$; if, also, the problem is regular, the forms

$$
f_{p^{i} p^{i} \xi^{i} \xi^{j}} \text { (if } \nu=1 \text { ) and } f_{p_{\alpha} p_{\beta}} \lambda_{\alpha} \lambda_{\beta} \text { (if } N=1 \text { ) }
$$

are required to be positive definite. The condition in (13) does not imply the convexity of $f$ in all the $p_{\alpha}^{i}$ taken together in the general case. However, in all regular cases, the Euler equations form a strongly elliptic system in the sense defined by Nirenberg [45].

Now we observe that the equations ( 7 ) form a special case of the equations

$$
\begin{gathered}
\int_{G}\left\{\zeta_{, \alpha}^{i}(x) A_{i}^{\alpha}[x, z(x), \nabla z(x)]+\zeta^{i}(x) B_{i}[x, z(x), \nabla z(x)]\right\} d x=0 \\
\forall \zeta \in C^{1}(\bar{G}) \ni \zeta=0 \quad \text { on } \partial G,
\end{gathered}
$$

where we have replaced $f_{p_{\alpha}}(x, z, p)$ by $A_{i}^{\alpha}(x, z, p)$ and $f_{z^{i}}$ by $B_{i}$. If $z \in C^{1}(\bar{G})$ and satisfies (15), we say that $z$ is a weak solution of the 
equations

$$
\frac{\partial}{\partial x^{\alpha}} A_{i}^{\alpha}=B_{i}, \quad i=1, \cdots, N .
$$

Of course we may consider equations of higher order and integrals involving derivatives of higher order. For that purpose we introduce the following notation: We let $\alpha=\left(\alpha_{1}, \cdots, \alpha_{\nu}\right)$, in which each $\alpha_{\lambda}$ is a nonnegative integer, be a multi-index, and denote

$$
|\alpha|=\alpha_{1}+\cdots+\alpha_{\nu}, \quad D^{\alpha} \phi=\frac{\partial^{|\alpha|} \phi}{\left(\partial x^{1}\right)^{\alpha_{1}} \cdots\left(\partial x^{\nu}\right)^{\alpha_{\nu}}},
$$

where $D^{\alpha} \phi=\phi$ if $\alpha=0$. Then, if

$$
\begin{aligned}
& I(z, G)=\int_{G} f[x, D z(x)] d x, \quad D z(x)=\left\{D^{\alpha} z^{i}(x)\right\}, \\
& 0 \leqq|\alpha| \leqq m_{i}, \quad i=1, \cdots, N,
\end{aligned}
$$

the first variation of $I$ is given by

$$
I_{1}(z, \zeta ; G)=\int \sum_{G=1}^{N} \sum_{|\alpha| \leqq m_{i}} D^{\alpha} \zeta^{i}(x) \cdot f_{p_{\alpha}^{i}}[x, D z(x)] d x .
$$

If each $z^{i} \in C^{m_{i}}(\bar{G})$ and $I_{1}(z, \zeta ; G)=0$ for all such $\zeta \ni D^{\alpha} \zeta^{i}=0$ on $\partial G$ for $0 \leqq|\alpha| \leqq m_{i}-1$, we say that $z$ is a weak solution of the equations

$$
\sum_{|\alpha| \leqq m_{i}}(-1)^{|\alpha|} D^{\alpha} f_{p_{\alpha}^{i}}=0 .
$$

We may replace $f_{p_{\alpha}}$ by $A_{i}^{\alpha}$ in (19) obtaining

$$
\int_{G} \sum_{i=1}^{N} \sum_{|\alpha| \leqq m_{i}} D^{\alpha} \zeta^{i} \cdot A_{i}^{\alpha}[x, D z(x)] d x=0 \quad \forall \zeta^{i} \in C_{0}^{m_{i}}(\bar{G}) .
$$

Finally, we may consider equations like (21) but in which $m_{i}$ is replaced by some integer $p_{i}$ and, in $A_{i}^{\alpha}, D z$ stands for $\left\{D^{\beta} z^{j}\right\}$ where $\alpha$ and $\beta$ satisfy proper inequalities. As an example of these more general equations, we give

$$
\begin{aligned}
\int_{G}\left\{\zeta_{, \alpha \beta}(x) a^{\alpha \beta}[x, z(x)]+\zeta_{, \alpha}(x) a^{\alpha}[x, z, \nabla z]\right. & \\
& \left.+\zeta a\left[x, z, \nabla z, \nabla^{2} z\right]\right\} d x=0 .
\end{aligned}
$$

In general we shall not discuss equations more general than (21). In all these cases the problem is to find conditions on the $A_{i}^{\alpha}$ in (21) which imply that any weak solution of (21) is a sufficiently differentiable solu- 
tion of the corresponding differential equations (or perhaps possesses some additional differentiability). Actually we shall often allow our weak solutions to be in properly chosen Sobolev spaces; we shall give more details later.

The general nature of the problem above is to conclude differentiability of solutions in addition to the minimum required to be solutions of the given equations. One of the earliest papers along this line was Hilbert's famous address [18] in which he proposed some 23 problems. Of these, the 19 th was to show that the regular solutions of any analytic regular variational problem are analytic and the 23rd was, vaguely, to develop a theory of the calculus of variations. A problem more general than the 19 th was solved by S. Bernstein in his famous memoir of 1904 [3] where he proved for the case of any analytic second order elliptic equation and solution of class $C^{3}$ on a domain in $R^{2}$ that the solution is analytic. His proof was rather long and many people presented simpler proofs, including $H$. Lewy [26], T. Rado [48], M. Gevrey [14], S. Bernstein himself [4], and others. E. E. Levi [25] proved the analyticity of the solutions of linear, analytic, elliptic equations of higher order in two dimensions. Bernstein's result was extended to solutions of class $C_{\mu}^{2}$ on domains in $R^{n}$ by E. Hopf [21] and was extended to higher order systems on $R^{n}$ by I. Petrovsky [47]. C. B. Morrey, Jr. and L. Nirenberg [40] showed that regular solutions of analytic linear elliptic systems satisfying analytic Dirichlet boundary data along an analytic portion of the boundary of a domain could be extended analytically across that portion. This result was extended to analytic nonlinear systems in $1957 / 58$ by A. Friedman [13] and myself [35], independently, using different methods. Finally, in my book [38], I treat the case of general regular boundary conditions (in the sense of Agmon, Douglis and Nirenberg [1]).

A somewhat different series of generalizations of Bernstein's result was begun by L. Lichtenstein [27] when he showed in 1912 that a solution of class $C^{2}$ on a domain in $R^{2}$ of an analytic variational problem (of the type in (1) with $\nu=2, N=1$ ) is analytic. This result was extended in 1929 by E. Hopf [20] to the case where the solution was required only to be of class $C_{\mu}^{1}$ (of class $C^{1}$ with derivatives satisfying Hölder conditions of exponent $\mu$ ) for some $\mu$ with $0<\mu<1$. Morrey [30] extended this result further in January 1938 to the case where the solution was required merely to satisfy a Lipschitz condition.

All of the results mentioned so far assumed the existence of a solution having certain differentiability properties. Hilbert [19] was probably the first to use variational methods to prove the existence 
of a harmonic function having given boundary values on a domain of a fairly general type.

Tonelli, Lebesgue, Fréchet, and many others developed the socalled "direct methods" of the calculus of variations. The idea of these methods is to show

(i) that the integral to be minimized is lower-semicontinuous with respect to some kind of convergence,

(ii) that it is bounded below in some class of "admissible functions," and

(iii) that there is a "minimizing sequence," i.e. a sequence for which the integral tends to its infimum, which converges in the sense required to some admissible function.

Tonelli exploited and popularized these methods in a series of papers and a book [56]-[62]. He applied the methods to many one dimensional (i.e. $\nu=1$ ) problems and to some two dimensional ones. For the one dimensional problems, he found it expedient to allow absolutely continuous functions as admissible and to use uniform convergence. This comes about roughly as follows: Suppose that

$$
f(x, z, p) \geqq m|p| r-K, \quad r>1, m>0,
$$

(which is not unreasonable since $f$ is convex in $p$ ). Then

$$
\int_{a}^{b}\left|z_{n}^{\prime}(x)\right| r d x \leqq L
$$

in any minimizing sequence $\left\{z_{n}\right\}$. From (24), one sees from the Hölder inequality that any minimizing sequence is equicontinuous. It is also true for any uniformly convergent subsequence of a minimizing sequence that the limit function is also absolutely continuous with

$$
\int_{a}^{b}\left|z^{\prime}(x)\right| r d x \leqq \liminf _{n \rightarrow \infty} \int_{a}^{b}\left|z_{n}^{\prime}(x)\right| r d x .
$$

(This type of convergence turns out to be weak convergence in the Sobolev-space $H_{r}^{1}(a, b)$ for the case where $\nu=1$.)

For the two dimensional problems, he defined functions which he called absolutely continuous but then found it expedient to require $f$ to satisfy (23) with $r>2$ (where $|p|=\left(p_{1}^{2}+p_{2}^{2}\right)^{1 / 2}$ ) in order to obtain equicontinuous minimizing sequences. Actually if he assumed that $f(x, z, p) \geqq f(x, z, 0)=0$ for all $(x, z, p)$, he could allow $r=2$. However, this leaves a gap, $1<r<2$, in which Tonelli was unable to obtain a general theorem. Moreover, if one considers integrals in which $\nu>2$, 
one soon finds that one must assume $r>\nu$ in order to assure that minimizing sequences are equicontinuous. To see this, it is only necessary to observe that the functions

$$
\log \log \left(1+|x|^{-1}\right), \quad|x|^{-h}, \quad 0<|x|<1
$$

are limits of $C^{1}$ functions $z_{n}$ in which

$$
\int_{G}\left|\nabla z_{n}\right|^{\nu} d x \text { and } \int_{G}\left|\nabla z_{n}\right|^{k} d x \quad \text { for } k<\nu /(h+1)
$$

are uniformly bounded over the unit ball $G$.

In order to get a more complete existence theory, the writer and Calkin [4], [31], [32], [33] found it expedient to allow as admissible, functions which are still more general than Tonelli's functions and to allow correspondingly more general types of convergence and boundary values. These new spaces of functions can now be identified with the so-called Sobolev spaces $H_{p}^{1}(G)$ (or $W_{p}^{1}(G)$ ) which are now used by many writers in many connections. Unfortunately the minimizing function shown to exist was known only to be in one of these general spaces and hence was not known to be continuous, let alone of class $C^{2}$ ! However, A. Haar [17] (see also T. Rado [49]) had proved previously that if $\nu=2, N=1$, and $f=f(p)$, there exists a unique minimizing function $z$ which is defined on a strictly convex domain $G$ and which satisfies a Lipschitz condition there, provided the given boundary values satisfy a certain "three point condition." Combining this with my January 1938 result mentioned above, we obtain an existence and differentiability theorem for the Euler equation of any analytic variational problem of this type. During the year $1937 / 38$, I was able to show for a wide class of integrals in the case $\nu=2, N$ arbitrary, that any minimizing vector satisfies a "Dirichlet growth" condition and that any solution satisfying this condition is of class $C_{\mu}^{n}(n \geqq 3,0<\mu$ $<1)$ if $f$ is and is analytic or $C^{\infty}$ if $f$ is. I lectured on this work in the seminar of Professor Marston Morse during the spring of 1938. Notes on these lectures were prepared by H. Busemann and are still to be found in the library of the Institute for Advanced Study under his name.

We must now define the so-called Sobolev spaces $H_{p}^{m}(G)$. Actually, these functions were used by G. C. Evans [11] in his work on potential theory beginning in 1920. Others had used these functions well before Sobolev [55] proved his famous result in 1938. Many of the standard results concerning such functions were proved in the papers by Calkin and Morrey cited above. Since distributions and partitions of unity had not been introduced, different terminology was used of course. 
Definition. A function $z$ is of class $H_{r}^{m}(G), r \geqq 1, G$ open, $\Leftrightarrow z$ is of class $L_{r}(G)$ and there exist functions $p_{\alpha}, 0 \leqq|\alpha| \leqq m$, each also of class $L_{r}(G)$ such that

$$
\int_{G} g(x) p_{\alpha}(x) d x=(-1)^{|\alpha|} \int_{G} z(x) D^{\alpha} g(x) d x
$$

for every $g \in C^{\infty}(G)\left(C_{c}^{\infty}\right.$, compact support on $\left.G\right)$.

It is clear that the functions $p_{\alpha}$ are uniquely determined up to null functions and that if $z$ is of class $H_{r}^{m}(G)$ and $z^{*}=z$ almost everywhere on $G$, then $z^{*}$ is of class $H_{r}^{m}(G)$ and the same functions $p_{\alpha}$ will do for $z^{*}$.

Definition. As in the case of the $L_{r}$ spaces, the elements of the space $H_{r}^{m}(G)$ are the classes of equivalent functions of class $H_{r}^{m}(G)$. We denote the classes of equivalent functions $p_{\alpha}$ by $z_{, \alpha}$ or $D^{\alpha} z$ and call them the distribution derivatives of the element $z$.

It turns out that $z \in H_{r}^{m}(G) \Leftrightarrow z$ and its distribution derivatives of order $\leqq m-1 \in H_{r}^{1}(G)$, etc. Of course we may regard an element $z \in H_{r}^{m}(G)$ as a distribution and then the distribution corresponding to $z, \alpha$ would be the corresponding derivative of $z$ in the distribution sense. The spaces $H_{r}^{m}(G)$ have been defined for all real $m$. We may allow real or complex functions $z$.

The following theorems about these spaces are known (see, for instance, [38, Chapter 3]).

The spaces $H_{r}^{m}(G)$ are Banach spaces if we define

$$
\|z\|_{r}^{m}=\sum_{|\alpha| \leq m}\|z, \alpha\|_{r}^{0}
$$

for instance. If $r=2$, they are Hilbert spaces if we define

$$
(z, w)^{m}=\sum_{|\alpha| \leqq m}(z, \alpha, w, \alpha)^{0} .
$$

If $r>1$, the spaces are reflexive.

If $z \in C^{m-1}(G)$ and all the $D^{\alpha} z$ with $|\alpha| \leqq m-1$ are locally Lipschitz on $G$, then $z$ is of class $H_{r}^{m}(G) \Leftrightarrow$ its norm (as defined in (26)) is bounded.

Functions of class $C^{\infty}(G)$ with finite norm in (26) are dense in $H_{r}^{m}(G)$; if $G$ is "strongly Lipschitz" (regular in the sense of Calderon) (see [38, p. 72]), the restrictions to $G$ of functions of class $C_{c}^{\infty}(D)$, where $D \supset \bar{G}$, are dense in $H_{r}^{m}(G)$.

Suppose $x=x(y)$ is a regular mapping of class $C^{m}(m \geqq 1)$ of $H$ onto $G$ in which all the derivatives of $x(y)$ and of the inverse mapping $y(x)$ are bounded. Then if $u$ is of class $H_{r}^{m}(G)$ and

$$
v(y)=u[x(y)]
$$


$v \in H_{r}^{m}(H)$ and the derivatives of $v$ are obtained as usual a.e.

Each element $u^{*} \in H_{r}^{1}(G)$ contains a representative $u$ which is absolutely continuous along almost all lines parallel to each axis and which is such that if $v$ is defined by (28), then $v$ has the same property and is, of course of class $H_{r}^{1}(H)$. If $u^{*}$ contains a continuous representative, then that is unique and can be taken as $u$.

(Sobolev [55].) If $G$ is Lipschitz (see [38, p. 77]) and $z \in H_{r}^{1}(G)$ with $1 \leqq r<\nu$, then $z \in L_{s}(G)$ and there exists a constant $C(\nu, r, G)$ such that

$$
\|z\|_{s}^{0} \leqq C \cdot\|z\|_{r}^{1}, \quad s=\nu r /(\nu-r) .
$$

Definition. We define the space $H_{r 0}^{m}(G)$ to be the closure in $H_{r}^{m}(G)$ of the set $C_{c}^{\infty}(G)$.

We have the following theorems.

(Poincaré's inequality.) Suppose $G \subset B\left(x_{0}, R\right)$ and $z \in H_{r 0}^{m}(G)$. Then

$$
\int_{G}\left|\nabla^{k} z(x)\right|^{r} d x \leqq r^{k-m} R^{(m-k) r} \int_{G}\left|\nabla^{m_{z}}(x)\right| r d x, \quad 0 \leqq k \leqq m .
$$

(a) Suppose $u \in H_{r 0}^{m}(G), V(x)=u(x)$ for $x \in G$, and $V(x)=0$ elsewhere. Then $V \in H_{r}^{m}\left(R_{\nu}\right)$ and $V \in H_{r 0}^{m}(\Delta)$ for any open $\Delta \supset G$. Moreover $D^{\alpha} V(x)=D^{\alpha} u(x)$ on $G$ and $D^{\alpha} V(x)=0$ for $x \in R_{\nu}-G$ if $0 \leqq|\alpha| \leqq m$ (a.e.).

(b) Suppose $u \in H_{r}^{m}(G), D \subset G, v \in H_{r}^{m}(D), v-\left.u\right|_{D} \in H_{r 0}^{m}(D), U(x)$ $=v(x)$ on $D$, and $U(x)=u(x)$ on $G-D$. Then $U \in H_{r}^{m}(G), U-u$ $\in H_{r 0}^{m}(G)$, and $D^{\alpha} U(x)=D^{\alpha} v(x)$ on $D$ and $D^{\alpha} U(x)=D^{\alpha} u(x)$ on $G-D$ (a.e.).

$z_{n} \rightarrow\left(\right.$ tends weakly) $z$ in $H_{r}^{m}(G) \Leftrightarrow D^{\alpha} z_{n} \rightarrow D^{\alpha} z$ in $L_{r}(G)$ for each $\alpha$ with $|\alpha| \leqq m$.

The first of these results shows that

$$
\|z\|_{r 0}^{m}=\left[\int_{G}\left|\nabla^{m} z\right|^{r} d x\right]^{1 / r}
$$

is equivalent to the norm $\|z\|_{r}^{m}$ for $z \in H_{r 0}^{m}(G)$.

The change of variable theorem enables us to define the spaces $H_{r}^{m}$ on manifolds of class $C^{m}$ (or even $C_{1}^{m-1}$ ); of course there are no particularly natural norms on these spaces although many satisfactory norms can be defined which are topologically equivalent if the manifold is compact. We have the following results.

Suppose $G$ is strongly Lipschitz and $m \geqq 1$. Then bounded subsets of $H_{r}^{m}(G)$ are conditionally sequentially compact as subsets of $H_{r}^{m-1}(G)$. If $u_{n} \rightarrow u$ in $H_{r}^{m}(G)$ then $u_{n} \rightarrow u$ in $H_{r}^{m-1}(G)$. If $m=1, G$ may be merely 
Lipschitz (see [38, p. 77]). The theorem above is true for any bounded domain if we replace the spaces $H_{r}^{m}(G)$ and $H_{r}^{m-1}(G)$, respectively by $H_{r 0}^{m}(G)$ and $H_{r 0}^{m-1}(G)$.

If $G$ is (bounded and) of class $C_{1}^{m-1}$ the functions $u \in C_{1}^{m-1}(\bar{G})$ are dense in any space $H_{r}^{m}(G)$ with $r \geqq 1$ and there is a bounded operator $B$ from $H_{r}^{m}(G)$ into $H_{r}^{m-1}(\partial G)$ such that $B u=\left.u\right|_{\partial G}$ whenever $u \in C_{1}^{m-1}(\bar{G})$. If $u_{n} \rightarrow u$ in $H_{r}^{m}(G), B u_{n} \rightarrow B u$ in $H_{r}^{m-1}(\partial G)$. If $r>1$, the mapping $B$ is compact.

We now present a sample lower-semicontinuity theorem. We need the fact that a convex function $f(\xi)$ satisfies

$$
f(\xi) \geqq f\left(\xi_{0}\right)+f_{, \alpha}\left(\xi_{0}\right)\left(\xi^{\alpha}-\xi_{0}^{\alpha}\right) \quad \forall \xi .
$$

Suppose $f=f(x, z, p)$ and the $f_{p_{\alpha}^{i}}$ are continuous with $f(x, z, p) \geqq 0$ for all $(x, z, p)$, suppose $f$ is convex in $p$ for each $(x, z)$, and suppose $z_{n} \rightarrow z$ in $H_{1}^{1}(D)$ for each $D \subset \subset G$. Then

$$
I(z, G) \leqq \lim \inf I\left(z_{n}, G\right) .
$$

Proof. Choose $D \subset \subset G$. The weak convergence in $H_{1}^{1}\left(D^{\prime}\right)$ for each $D^{\prime} \subset \subset G$ implies the strong convergence of $z_{n}$ to $z$ in $L_{1}(D)$. By choosing a subsequence, still called $z_{n}$, in which $I\left(z_{n}, G\right) \rightarrow$ its former $\lim$ inf, we may assume that $z_{n}(x) \rightarrow z(x)$ a.e. on $D$. We now suppose $I(z, D)$ $<+\infty$. Then, for each $\epsilon>0$, there is a compact set $S \subset D$ on which representatives of $z$ and $\nabla z$ are continuous, on which $z_{n}$ converges uniformly to $z$, and which is such that

$$
I(z, S)>I(z, D)-\epsilon
$$

(if $I(z, D)=+\infty$, we may take $I(z, S)>M$, arbitrary). Then from the convexity of $f$, we conclude that, for $x \in S$,

$$
\begin{aligned}
f\left[x, z_{n}(x), \nabla z_{n}(x)\right] \geqq & f\left[x, z_{n}(x), \nabla z(x)\right]+f_{p}[x, z(x), \nabla z(x)] \\
& \cdot\left[\nabla z_{n}(x)-\nabla z(x)\right] \\
& +\left\{f_{p}\left[x, z_{n}(x), \nabla z(x)\right]-f_{p}[x, z(x), \nabla z(x)]\right\} \\
& \cdot\left[\nabla z_{n}(x)-\nabla z(x)\right] .
\end{aligned}
$$

The weak convergence implies the weak convergence of $\nabla z_{n}$ to $\nabla z$ in $L_{1}(S)$ which implies, in turn, that

$$
\int_{S} f_{p}[x, z(x), \nabla z(x)] \cdot\left[\nabla z_{n}(x)-\nabla z(x)\right] d x \rightarrow 0 .
$$

The uniform convergence of $z_{n}$ to $z$ on $S$ and the uniform boundedness of the $L_{1}(S)$ norms of $\nabla z_{n}$ and $\nabla z$ implies that 


$$
\int_{S}\left\{f_{p}\left[x, z_{n}, \nabla z\right]-f_{p}[x, z, \nabla z]\right\} \cdot\left[\nabla z_{n}-\nabla z\right] d x \rightarrow 0
$$

Hence

$$
I(z, D)-\epsilon(\text { or } M) \leqq \lim _{n \rightarrow \infty} \int_{S} f\left[x, z_{n}, \nabla z\right] d x \leqq \lim \inf I\left(z_{n}, G\right) .
$$

We now present a sample existence theorem.

Suppose that

(i) $f$ and $f_{p_{\alpha}^{i}}$ are continuous in their arguments;

(ii) $f$ is convex in $p$ for each $(x, z)$;

(iii) for all $(x, z, p)$

$$
f(x, z, p) \geqq m|p|^{k}, \quad m>0, \quad k>1 ;
$$

(iv) $F^{*}$ is a nonempty family of vector functions which is compact with respect to weak convergence in $H_{k}^{1}(G)$;

(v) $F$ is a family, closed under weak convergence in $H_{k}^{1}(G)$, such that each $z$ in $F$ coincides with some $z^{*}$ in $F^{*}$ on $\partial G\left(\right.$ i.e. $\left.z-z^{*} \in H_{k 0}^{1}(G)\right)$;

(vi) $I\left(z_{0}, G\right)<+\infty$ for some $z_{0} \in F$; and

(vii) $G$ is bounded.

Then $I(z, G)$ takes on its minimum for some $z$ in $F$.

Since we have not made any assumptions about $G$ other than that it is bounded and since the admissible functions are not necessarily continuous, the most convenient way to specify the boundary values of a function $z$ is to state that $z-z^{*} \in H_{k 0}^{1}(G)$ for some given $z^{*}$. Thus the family $F^{*}$ defines the boundary values being allowed, so to speak. Of course $F^{*}$ could consist of a single function $z^{*}$. In case $G$ is at least Lipschitz, then the family $F^{*}$ could be replaced by a family of functions defined on $\partial G$ and then each $z$ in $F$ would be required to be such that $B z \in F^{*}$. This change would make little difference in the proof below if $F^{*}$ were closed in $L_{k}(\partial G)$.

Proof. Let $\left\{z_{n}\right\}$ be a minimizing sequence; we may assume that $I\left(z_{n}, G\right) \leqq I\left(z_{0}, G\right)=M$ for every $n$. Using (iii), we find that

$$
\int_{G}\left|\nabla z_{n}\right|^{k} d x \leqq L=M / m, \quad n=1,2, \cdots
$$

From (v), we know that there exists a $z_{n}^{*} \in F^{*} \ni w_{n}=z_{n}-z_{n}^{*} \in H_{k 0}^{1}(G)$ for each $n$. From (iv), we may extract a subsequence, still called $\{n\}$ such that $z_{n}^{*} \rightarrow$ some $z^{*} \in F^{*}$. We also see that $\int_{G}\left|\nabla w_{n}\right|{ }^{k} d x$ and hence (since $\left.w_{n} \in H_{k 0}^{1}(G)\right)\left\|w_{n}\right\|_{k}^{1}$ is uniformly bounded. Hence, from the reflexivity we conclude that, for a further subsequence, still called 
$\{n\}, w_{n} \neg w$ in $H_{k}^{1}(G)$. Since $H_{k 0}^{1}(G)$ is a closed linear manifold in $H_{k}^{1}(G)$ we see that $w \in H_{k 0}^{1}(G)$. Thus $z_{n} \rightarrow z=z^{*}+w$ and $z$ is the desired solution.

If $f$ satisfies no additional conditions, it is not necessarily true that a minimizing function has any further continuity and differentiability properties. Rather than stating the most general conditions ensuring further differentiability, we state two sets of conditions on the integrand function $f$ under which differentiability results have been obtained. We require $f$ first to satisfy the

Common condition. $f \in C_{\mu}^{2}$ in its arguments or $f$ and $f_{p} \in C_{\mu}^{n-1}$ for some $n \geqq 3$ and some $\mu$ with $0<\mu<1$.

Besides this condition, we require that $f$ satisfy one of the following sets for all $(x, z, p)$ :

$$
\begin{aligned}
& m V^{k}-K \leqq f(x, z, p) \leqq M V^{k} \\
& \left|f_{p}\right|^{2}+\left|f_{p x}\right|^{2}+\left|f_{z}\right|^{2}+\left|f_{z x}\right|^{2} \leqq M_{1}^{2} V^{2 k-2}
\end{aligned}
$$

(A) $\left|f_{p z}\right|^{2}+\left|f_{p p}\right|^{2} \leqq M_{1}^{2} V^{2 k-4}$ $m_{1} V^{k-2}|\pi|^{2} \leqq \sum f_{p_{\alpha}^{i} j}^{i j \pi_{\alpha}^{i} \pi_{\beta}^{j}}$

$k>1, \quad 0<m \leqq M, \quad 0<m_{1} \leqq M_{1}, \quad V=\left(1+|z|^{2}+|p|^{2}\right)^{1 / 2}$ $\left(\left|f_{p p}\right|^{2}=\sum\left(f_{p_{\alpha}^{i} p_{\beta}^{j}}\right)^{2}, \quad\left|f_{p z}\right|^{2}=\sum\left(f_{\left.p_{\alpha}^{i} j_{j}\right)^{2}}, \quad|\pi|^{2}=\sum\left(\pi_{\alpha}^{i}\right)^{2}\right.\right.$, etc.

(A') Same as (A) except that $f=f(x, p), V=\left(1+|p|^{2}\right)^{1 / 2}$.

$$
\begin{aligned}
& m V^{k}-K \leqq f(x, z, p) \leqq M V^{k} \\
& \left|f_{z}\right|^{2}+\left|f_{z x}\right|^{2}+\left|f_{z z}\right|^{2} \leqq M^{2}(R) V^{2 k} \\
& \left|f_{p}\right|^{2}+\left|f_{p z}\right|^{2}+\left|f_{p x}\right|^{2} \leqq M^{2}(R) V^{2 k-2} \\
& m_{1}(R) V^{k-2}|\pi|^{2} \leqq \sum f_{p_{\alpha}^{i} p_{\beta}^{j} \pi_{\alpha} \pi_{\beta}^{j} \leqq M_{1}(R) V^{k-2}|\pi|^{2}} \\
& k \geqq \nu, \quad 0<m \leqq M, \quad V=\left(1+|p|^{2}\right)^{1 / 2} \\
& 0<m_{1}(R) \leqq M_{1}(R) \quad \text { for }|x|^{2}+|z|^{2} \leqq R^{2} .
\end{aligned}
$$

We notice that (B) reduces to $\left(\mathrm{A}^{\prime}\right)$ if $f$ does not depend on $z$, except for the $R$-condition which is somewhat meaningless since we always assume $G$ bounded. To see the difference between (A) and (B), we notice that the $f$ defined by

$$
f(x, z, p)=\left[1+a_{i j}^{\alpha \beta}(x, z) p_{\alpha}^{i} p_{\beta}^{j}\right]^{k / 2}
$$


satisfies the conditions (B) but not (A) if $k \geqq \nu$ and the $a$ 's $\in C_{\mu}^{2}$ or $C_{\mu}^{n-1}$ if $n \geqq 3$ and the quadratic form is positive definite. We think of $k$ as the degree of $f$ in $(p, z)$ or $p$.

The first step in the proof of differentiability of the solutions is to note the following continuity properties of the solutions:

If $k \geqq \nu$, the minimizing functions are Hölder continuous on interior domains.

Proof. If $k>\nu$, we see by the Hölder inequality that

$$
\int_{B\left(x_{0}, r\right)}|\nabla z| d x \leqq C(\nu, k)\|\nabla z\|_{k}^{0} r^{\nu-1+\mu}, \mu=1-\nu / k, B\left(x_{0}, r\right) \subset G .
$$

If $\nu=k$ and $z$ is minimizing, we see from either (A) or (B) that

$$
m|p|^{k}-K^{\prime} \leqq f(x, z, p) \leqq M|p|^{k}+L^{\prime}, \quad 0<m \leqq M
$$

so that if $z$ is minimizing

$$
\begin{aligned}
& m \int_{B\left(x_{0}, r\right)}|\nabla z|^{\nu} d x-K^{\prime} \gamma_{\nu} r^{\nu} \leqq I\left[z, B\left(x_{0}, r\right)\right] \leqq I\left[Z, B\left(x_{0}, r\right)\right] \\
& \leqq L_{\gamma \nu}^{\prime} r^{\nu}+M \int_{B\left(x_{0}, r\right)}|\nabla Z|^{k} d x \\
&(\gamma \nu=\operatorname{meas} B(0,1))
\end{aligned}
$$

where $Z$ is any vector function such that $Z-z \in H_{k}^{1}\left[B\left(x_{0}, r\right)\right]$. Setting

$$
Z(s, \theta)=\bar{z}+(s / r)[z(r, \theta)-\bar{z}], \quad \theta \in \partial B(0,1)
$$

$$
\left(\bar{z}=\operatorname{av} z \text { on } \partial B\left(x_{0}, r\right)\right)
$$

it is easy to see, using the fact that

$$
|\nabla Z(s, \theta)|^{2}=Z_{s}^{2}+s^{-2}\left|\nabla_{\theta} Z\right|^{2}=r^{-2}\left\{|z(r, \theta)-\bar{z}|^{2}+\left|\nabla_{\theta} z(r, \theta)\right|^{2}\right\} \text {, }
$$

that (setting $d \sum$ as $(\nu-1)$-area element on $\partial B(0,1)$ )

$$
\begin{aligned}
\phi(r) \equiv & \int_{B\left(x_{0}, r\right)}|\nabla z|^{\nu} d x \leqq m^{-1} \gamma_{\nu}\left(K^{\prime}+L^{\prime}\right) r^{\nu} \\
& +C \int_{\partial B\left(x_{0}, r\right)}\left|\nabla_{\theta} z(r, \theta)\right|^{\nu} d \sum \leqq C_{1} r \phi^{\prime}(r)+C_{2} r^{\nu}
\end{aligned}
$$

It follows easily from (33) that

$$
\begin{aligned}
& \int_{B\left(x_{0}, r\right)}|\nabla z|^{\nu} d x \leqq\left(\phi(R)+C_{3} R^{\nu}\right)(r / R)^{\nu \mu} \\
& 0 \leqq r \leqq R, B\left(x_{0}, R\right) \subset G,
\end{aligned}
$$


from which (29) follows easily where, however, $C$ depends on the distance $d\left(x_{0}, \partial G\right)$ of $x_{0}$ from $\partial G$ and $\mu$ is that in (34). The result follows from the following theorem about Sobolev spaces.

If $z$ satisfies a condition like (29) where $C$ may depend on $d\left(x_{0}, \partial G\right)$ and $\mu$ is any number with $0<\mu<1$, then $z \in C_{\mu}^{0}(\bar{D})$ on any compact set $\bar{D} \subset G$ (see $[38, p .79])$.

The next step in the differentiability program is stated in the following theorem.

Suppose $f$ satisfies (A) or $\left(\mathrm{A}^{\prime}\right)$ for some $k>2$ or satisfies (B) with $k>\nu$. Then $I(z) \equiv I(z, G)$ is of class $C^{2}$ over the space $H_{k}^{1}(G)$ ( $G$ bounded). If $f$ satisfies (A) or $\left(\mathrm{A}^{\prime}\right)$ with $1<k \leqq 2$, then $I(z)$ is of class $C^{1}$ over $H_{k}^{1}(G)$. In all cases, if $z$ is minimizing, the first differential (variation) of $I$ vanishes at $z$.

In other words, if $z$ is minimizing $z$ satisfies (7). We call any function $z$ for which the first variation vanishes an extremal whether $z$ is minimizing or not and we also call such a $z$ a weak solution of the corresponding Euler equations.

So far, we have shown the existence only of a minimizing function for $I$ but this was done for rather general functions $f$. Recently Palais and Smale [46] have found a modification of the Morse theory which is applicable to a wide variety of variational problems. In their theory an extremal is just a critical point for the integral.

To illustrate the next step in the differentiability program, we sketch the proof of our January 1938 result mentioned above in which we assume that the extremal $z$ satisfies a Lipschitz condition and that $f=f(p, q)$, that $\nu=2$, that $f \in C_{\mu}^{2}$, and $f$ is regular (cf. the middle inequality in $\mathrm{A}$ or $\mathrm{B})$, i.e. that

$$
f_{p p}(p, q) \lambda^{2}+2 f_{p q} \lambda \mu+f_{q q} \mu^{2}>0 \quad \text { if } \lambda^{2}+\mu^{2}>0 .
$$

Then $z$ satisfies

$$
\begin{aligned}
\iint_{G}\left[\zeta_{x}(x, y) A(x, y)+\zeta_{y}(x, y) B(x, y)\right] d x d y= & 0 \\
& \forall \zeta \in \operatorname{Lip}_{c}(G) \\
A(x, y)=f_{p}\left[z_{x}(x, y), z_{y}(x, y)\right], \quad B(x, y)= & f_{q}[\text { same }] .
\end{aligned}
$$

Choose $\zeta \in \operatorname{Lip}_{c}(G)$ and define (for $h$ small, $\neq 0$ )

$$
\begin{aligned}
& \zeta_{h}(x, y)=h^{-1}[\zeta(x-h, y)-\zeta(x, y)], \\
& z_{h}(x, y)=h^{-1}[z(x+h, y)-z(x, y)] .
\end{aligned}
$$


If we replace $\zeta$ in (10) by $\zeta_{h}$, make the obvious change of variable in the terms involving $\zeta_{x}(x-h, y)$, etc., we obtain the equation

$$
h^{-1} \iint_{G}\left(\zeta_{x} \Delta A+\zeta_{y} \Delta B\right) d x d y=0, \Delta A=A(x+h, y)-A(x, y) .
$$

Since $z$ is Lipschitz, its partial derivatives exist almost everywhere and are bounded. Thus, for almost all $(x, y)$, we may express $\Delta A$ and $\Delta B$ in terms of $z_{h x}$ and $z_{h y}$ using the integral form of the theorem of the mean. When this is done, (38) becomes

$$
\iint_{G}\left[\zeta_{x}\left(a_{h} z_{h x}+b_{h} z_{h y}\right)+\zeta_{y}\left(b_{h} z_{h x}+c_{h} z_{h y}\right)\right] d x d y=0
$$

where $a_{h}, b_{h}, c_{h}$ are bounded and measurable independently of $h$ and

$$
a_{h}(x, y)=\int_{0}^{1} f_{p p}\left[z_{x}(x, y)+t \Delta z_{x}, z_{y}(x, y)+t \Delta z_{y}\right] d t
$$

and $b_{h}$ and $c_{h}$ are obtained similarly from $f_{p q}$ and $f_{q q}$. Also, from (35) and the boundedness of $z_{x}$ and $z_{y}$, we conclude that there are numbers $m$ and $M$, independent of $h$, such that

$$
m\left(\lambda^{2}+\mu^{2}\right) \leqq a_{h} \lambda^{2}+2 b_{h} \lambda \mu+c_{h} \mu^{2} \leqq M\left(\lambda^{2}+\mu^{2}\right), \quad 0<m \leqq M .
$$

Now, we choose $\delta>0$ and define

$$
\begin{array}{rlrl}
\phi(x, y) & =1, & & (x, y) \in G_{2 \delta}, \\
& =1-\delta^{-1} d\left(x, y, G_{2 \delta}\right), & & (x, y) \in G_{\delta}-G_{2 \delta}, \\
& =0, & & (x, y) \in G-G_{\delta}, \\
G_{a} & =\{(x, y) \mid B(x, y ; a) \subset G\} & \\
\zeta & =\phi Z_{h}, & Z_{h}=\phi z_{h}, & 0<|h|<\delta .
\end{array}
$$

Then, for each $h, \zeta$ and $Z_{h}$ are Lipschitz and have support on $\bar{G}_{\delta}$. We also have (a.e.)

$$
\begin{aligned}
\zeta_{x} & =\phi\left(Z_{h x}+\phi_{x} z_{h}\right), & \zeta_{y} & =\phi\left(Z_{h y}+\phi_{y} z_{h}\right), \\
\phi z_{h x} & =Z_{h x}-\phi_{x} z_{h}, & \phi z_{h y} & =Z_{h y}-\phi_{y} z_{h .} .
\end{aligned}
$$

Substituting these results into (39), we find that

$$
\begin{aligned}
\iint_{G_{\delta}}\left[a_{h} Z_{h x}^{2}+2 b_{h} Z_{h x} Z_{h y}+c_{h} Z_{h y}^{2}\right) & \\
& \left.-z_{h}^{2}\left(a_{h} \phi_{x}^{2}+2 b_{h} \phi_{x} \phi_{y}+c_{h} \phi_{y}^{2}\right)\right] d x d y=0 .
\end{aligned}
$$

From (41) and the definitions of $\phi$ and $Z_{h}$, we see that 


$$
\iint_{G_{2 \delta}}\left(z_{h x}^{2}+z_{h y}^{2}\right) d x d y \leqq \frac{M}{m} \delta^{-2} \iint_{G_{\delta}} z_{h}^{2} d x d y .
$$

Since $z$ is Lipschitz, the right side of (42) is bounded independently of $h$ for each $\delta>0$ so the left side is also. Thus, for a sequence of $h \rightarrow 0$, $z_{h x} \rightarrow u$ and $z_{h y} \rightarrow v$ in $L_{2}\left(G_{2 \delta}\right)$ for some $u$ and $v$. But now $z_{h}(x, y) \rightarrow z_{x}(x, y)$ $=p(x, y)$ a.e. and boundedly. Now if $\psi \in C_{c}^{\infty}\left(G_{2 \delta}\right)$

$$
\begin{aligned}
& \iint_{G_{2 \delta}} \psi z_{h x} d x d y=-\iint_{G_{2 \delta}} \psi_{x} z_{h} d x d y, \\
& \iint_{G} \psi z_{h y} d x d y=-\iint_{G} \psi_{y} z_{h} d x d y .
\end{aligned}
$$

We may let $h \rightarrow 0$ in (43) (thru the special subsequence) and get

$$
\begin{aligned}
& \iint_{G_{2 \delta}} \psi u d x d y=-\iint_{G_{2 \delta}} \psi_{x} p d x d y, \\
& \iint_{G_{2 \delta}} \psi v d x d y=-\iint_{G_{2 \delta}} \psi_{\nu} p d x d y .
\end{aligned}
$$

Thus $p \in H_{2}^{1}\left(G_{2 \delta}\right)$ and $u=p_{x}, v=p_{y}$. Since $a_{h}, b_{h}, c_{h}$ tend a.e. and boundedly to their respective limits

$$
a=f_{p q}\left[z_{x}(x, y), z_{y}(x, y)\right], \quad b=f_{p q}[], \quad c=f_{q q}[]
$$

$\zeta_{x} a_{h} \rightarrow \zeta_{x} a$, etc., strongly in $L_{2}\left(G_{2 \delta}\right)$ if $\zeta \in \operatorname{Lip}_{c}\left(G_{2 \delta}\right)$. Thus we may let $h \rightarrow 0$ in (39) and conclude that $p$ satisfies the limiting equations

$$
\iint_{G_{2 \delta}}\left[\zeta_{x}\left(a u_{x}+b u_{y}\right)+\zeta_{y}\left(b u_{x}+c u_{y}\right)\right] d x d y=0 .
$$

Likewise $q=z_{y}$ is seen to $\in H_{2}^{1}\left(G_{2 \delta}\right)$ and to satisfy (46).

Now, I proved before the war that solutions of such equations are Hölder continuous on interior regions if $\nu=2$. Using that information, we have $p$ and $q$ satisfying the limit equations (46) in which $a, b$, and $c$ are Hölder continuous. From an old theorem of Lichtenstein, it follows that $p$ and $q \in C_{\mu}^{1}$ so that $z \in C_{\mu}^{2}$. Higher differentiability follows by repetition.

As was mentioned above the author carried the program above through for the cases where $f$ satisfies the conditions (B) with $k=\nu=2$ and $N$ arbitrary during the year $1937 / 38$. The author reported on this in an invited address to the Society at its meeting in Pasadena on December 2, 1939 [32]. The long manuscript for this work was approved for publication by the University of California Press in 
1939 and appeared in December 1943 [33]. The writer used these results in his solution of the problem of Plateau on a Riemannian manifold [34].

Attempts to extend these results to (nonlinear) cases where $\nu>2$ met with no success until De Giorgi [9] and Nash [42] independently showed that a solution $u$ in $H_{2}^{1}(G)$ of an equation (like (46)) of the form

$$
\begin{gathered}
\int_{D} \zeta_{, \alpha} a^{\alpha \beta} u_{, \beta} d x=0, \\
\forall \zeta \in H_{20}^{1}(D), \quad m|\lambda|^{2} \leqq a^{\alpha \beta}(x) \lambda_{\alpha} \lambda_{\beta} \leqq M|\lambda|^{2},
\end{gathered}
$$

with the $a^{\alpha \beta}$ bounded and measurable, is Hölder continuous on interior domains. A simplification of this work due to Moser [41] and the development of other techniques enabled the author and a student E. R. Buley [36], [37] and simultaneously O. A. Ladyzenskaya and N. Uraltseva [22], [23], [24] to prove that the solutions (of a variational problem) $\in C_{\mu}^{n}$ in case the integrand function $f$ satisfies the conditions (A) or ( $\left.\mathrm{A}^{\prime}\right)$ with $N=1$ and $k>1$ or (B) with $k \geqq \nu$ and $N=1$, but $\nu$ arbitrary in both cases. Ladyzenskaya and Uraltseva obtained also the differentiability results in the cases (B) where $2 \leqq k<\nu$, provided the solution was known to be bounded. They also noticed that the results could be carried over to (weak) solutions $z$ in $H_{k}^{1}(G)$ of equations of the form

$$
\int_{G}\left[\zeta, \alpha A^{\alpha}(x, z, \nabla z)+\zeta B(x, z, \nabla z)\right] d x=0 \quad \forall \zeta \in \operatorname{Lip}_{c} G
$$

provided the $A^{\alpha}(x, z, p)$ and $B(x, z, p)$ satisfy conditions analogous to $(\mathrm{A}),\left(\mathrm{A}^{\prime}\right)$, and $(\mathrm{B})$, namely,

$$
\begin{aligned}
& |A|^{2}+\left|A_{x}\right|^{2}+|B|^{2}+\left|B_{x}\right|^{2} \leqq M_{1}^{2} V^{2 k-2} \\
& \left|A_{z}\right|^{2}+\left|A_{p}\right|^{2}+\left|B_{z}\right|^{2}+\left|B_{p}\right|^{2} \leqq M_{1}^{2} V^{2 k-4} \\
& m_{1} V^{k-2}|\pi|^{2} \leqq A_{i p_{\beta}^{j} \pi_{\alpha}^{i} \pi_{\beta}^{j}}^{\alpha} \\
& k>1, \quad m_{1}>0, \quad V=\left(1+|z|^{2}+|p|^{2}\right)^{1 / 2}
\end{aligned}
$$

(C') The same as (C) with $A=A(x, p), B=0, V\left(1+|p|^{2}\right)^{1 / 2}, k>1$.

$$
\begin{aligned}
& |B|^{2}+\left|B_{z}\right|^{2}+\left|B_{x}\right|^{2} \leqq M_{1}^{2}(R) V^{2 k} \\
& |A|^{2}+\left|A_{z}\right|^{2}+\left|A_{x}\right|^{2}+\left|B_{p}\right|^{2} \leqq M_{1}^{2}(R) V^{2 k-2} \\
& m_{1}(R) V^{k-2}|\pi|^{2} \leqq A_{i p_{\beta} \pi_{\alpha} \pi_{\alpha} \pi_{\beta}, \quad\left|A_{p}\right| \leqq M_{1}(R) V^{k-2}} \\
& k \geqq \nu, \quad m_{1}(R)>0, \quad V=\left(1+|p|^{2}\right)^{1 / 2}, \quad|x|^{2}+|z|^{2} \leqq R .
\end{aligned}
$$


In all cases, it is assumed that the $A_{i}^{\alpha}$ and $B_{i} \in C_{\mu}^{1}$ if $n=2$ or the $A_{i}^{\alpha} \in C_{\mu}^{n-1}$ and $B_{i} \in C_{\mu}^{n-2}$ if $n \geqq 3$. And, of course, it is not assumed that

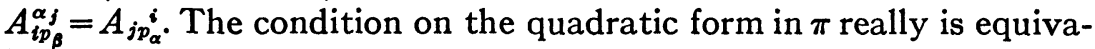
lent to a condition on its symmetric part

$$
\frac{1}{2}\left(A_{i p_{\beta}^{j}}^{\alpha}+A_{j p_{\alpha}^{i}}^{\beta}\right) \pi_{\alpha}^{i j} \pi_{\beta}^{j} \geqq m_{1}(R) V^{k-2}|\pi|^{2} .
$$

It is to be noticed that the results above for the cases $\nu>2$ all require $N=1$.

So far, I have spoken only of variational problems in nonparametric form. It will be recalled that an integral in parametric form is one in which the integral is invariant under diffeomorphisms (possibly with positive jacobian) in the independent variables. Thus one looks for solutions which are "geometric surfaces" in some sense, each of which might have many parametric representations which might be vector functions giving the same value to the integral. It turns out that the function $f$ for such a problem must be independent of $x$, must have $N \geqq \nu$, and must depend only on the $\nu \times \nu$ determinants in the $\left\{p_{\alpha}^{i}\right\}$ matrix. The area integral in (4) has this property. Such functions cannot be convex in the $p_{\alpha}^{i}$ although they do satisfy the condition (13) if the rank of the $p$ matrix is $\nu$. Also, in general, $f$ is singular along the manifold in the $p$-space where the $p$ matrix is of rank $<\nu$.

The problem of Plateau (the problem of finding a smooth minimal surface of least area) for surfaces which are images of the unit disc and bounded by a single given contour was solved in 1930/31 simultaneously by J. Douglas [10] and T. Rado [50]. These men and others, including McShane [29] and Courant [7] solved the problem in the 1930's for surfaces of more general topological types and/or bounded by more than one Jordan curve. The notion of surface used was that of a Fréchet surface (see, for instance, [38, pp. 350-352]) with specified topological type. In the early 1950's an existence theorem was proved independently by Cesari [6], Danskin [8], and Sigalov [54] for essentially the most general type of parametric problem with $\nu=2$ using Fréchet surfaces. In all these cases, use of conformal maps of the surfaces essentially reduced the problem to a nonparametric one; the area integral was replaced by the Dirichlet integral, since

$$
\left(\frac{E+G}{2}\right)^{2}=\left(\frac{E-G}{2}\right)^{2}+F^{2}+\left(E G-F^{2}\right) .
$$

The nonexistence of special maps of higher dimensional manifolds held up the solution of the problem of Plateau in higher dimensions until that of Reifenberg in 1960 [51 ], [52 ], [53]. He showed the existence of a compact point set of minimum Hausdorff $\nu$-measure among all such in $R^{N}$ which are bounded in a certain topological sense by a 
given compact set and showed that the resulting set consisted of the union of an open analytic manifold and a locally compact set of $\nu$-Hausdorff measure zero; in general, this singular set will not be empty since any analytic set is minimal. Very recently, Almgren [2] extended Reifenberg's results to a wide class of parametric problems in higher dimensions using the notions of currents, developed recently by Federer and Fleming [12] and his own notion of a varifold.

Also during the last five years, a theory of "monotone" operators has been developed and generalized by Minty, Browder, Visik, Lions, Leray, and others to yield existence theorems for (weak) solutions of quite general elliptic systems of the form

$$
\int \sum_{i=1}^{N} \sum_{|\alpha| \leqq m_{i}} D^{\alpha} \zeta^{i} \cdot A_{i}^{\alpha}[x, D z(x)] d x=0 \quad \forall \zeta^{i} \in C_{c}^{m_{i}}(G) .
$$

About a year ago, the author's paper Partial regularity results for non-Linear elliptic systems appeared in the Journal of Mathematics and Mechanics [39]. In that paper, I showed that if each $z^{i} \in H_{k}^{m_{i}}(G)$ for some $k>1$ and $m_{i} \geqq 1$, and if $z$ is a solution of (21), then $G=D \cup Z$, where $D$ is open and $Z$ is locally compact and of measure zero and each $z^{i} \in C^{m_{i}+2}(D)$ provided the $A_{i}^{\alpha}$ satisfy the conditions below. Additional differentiability of the $z^{i}$ follows from that of the $A_{i}^{\alpha}$.

The methods used in the proof of that result were very different from those illustrated above and are too technical to present here. The principal new method was an adaptation of one due to Almgren. We assume that the $A_{i}^{\alpha}(x, p)\left(p=\left\{p_{\beta}^{j}\right\}\right.$ where $\beta$ is a multi-index with $\left.0 \leqq|\beta| \leqq m_{j}\right)$ are of class $C_{\mu}^{2}$ for all $(x, p)$ and satisfy the following conditions:

$$
\begin{aligned}
& |A(x, p)|,\left|A_{x}(x, p)\right| \leqq M V^{k-1}, \quad\left|A_{p}\right|,\left|A_{p x}\right|,\left|A_{p p}\right| \leqq M V^{k-2} \\
& \sum_{i, j=1}^{N} \sum_{|\alpha|=m_{i}} \sum_{|\beta|=m_{j}} A_{i p_{\beta}^{j}}^{\alpha}(x, p) \pi_{\alpha}^{i} \pi_{\beta}^{j} \geqq m^{*} V^{k-2}|\pi|^{2}, \quad m^{*}>0, \\
& V^{2}=1+\sum_{i} \sum_{|\alpha| \leq m_{i}}\left(z_{, \alpha}^{i}\right)^{2}, \quad k \geqq 2 .
\end{aligned}
$$

I and a student have extended these results slightly.

Also, about a year ago, De Giorgi produced an example to show that the De Giorgi-Nash-Moser results do not carry over to the cases $N>1$ and E. Giusti and M. Miranda [16] exhibited an analytic variational problem in which $N \geqq 3$ and $\nu \geqq 3$ and in which $z=|x|^{-1} x$ is the unique solution having those boundary values on $\partial B(0,1)$. Thus it appears that, in general, singularities are to be expected in the solutions of variational problems when $N>1$. However, J. Neças [43], 
[44] has proved regularity for solutions of certain systems including some of higher order, in cases where $\nu=2$ and $k$ and $N$ arbitrary. There is also a paper by E. Giusti [15] in which optimal regularity holds except on a singular set of $(\nu-1)$ dimension zero. It would seem that a continuation of research in regularity theory will have to consist in proving theorems about the singular set; this would appear to require different techniques from those used until now.

\section{BiBLIOGRAPHY}

1. S. Agmon, A. Douglis, and L. Nirenberg, Estimates near the boundary for the solutions of elliptic differential equations satisfying general boundary values. II, Comm. Pure Appl. Math. 17 (1964), 35-92.

2. F. J. Almgren, Jr., Existence and regularity almost everywhere of solutions to elliptic variational problems among surfaces of varying topological type and singularity structure, Ann. of Math. 87 (1968), 321-391.

3. S. Bernstein, Sur la natur analytique des solutions des équations aux derivées partielles $d u$ second ordre, Math. Ann. 59 (1904), 20-76.

4. - Demonstration du théorème de $M$. Hilbert sur la natur analytique des solutions des équations du type elliptique san l'impoi des séries normales, Math. Z. 28 (1928), 330-348.

5. J. W. Calkin, Functions of several variables and absolute continuity. I, Duke Math. J. 6 (1940), 170-185.

6. L. Cesari, An existence theorem of the calculus of variations for integrals on parametric surfaces, Amer. J. Math. 74 (1952), 265-292.

7. R. Courant, Plateau's problem and Dirichlet's principle, Ann. of Math. 38 (1937), 679-724.

8. J. M. Danskin, On the existence of minimizing surfaces in parametric double integral problems in the calculus of variations, Riv. Mat. Univ. Parma 3 (1952), 43-63.

9. E. De Giorgi, Sulla differenziabilita e analiticita delle estremali degli integrali multipli regolari, Mem. Accad. Sci. Torino Cl. Sci. Fis. Mat. Nat. (3) 3 (1957), 25-43.

10. J. Douglas, Solution of the problem of Plateau, Trans. Amer. Math. Soc. 33 (1931), 263-321.

11. G. C. Evans, Fundamental points of potential theory, Rice Inst. Pamphlets, no. 7, Rice Institute, Houston, Tex., 1920, pp. 252-359.

12. H. Federer and W. H. Fleming, Normal and integral currents, Ann. of Math. (2) 72 (1960), 458-520.

13. A. Friedman, On the regularity of the solutions of nonlinear elliptic and parabolic systems of partial differential equations, J. Math. Mech. 7 (1958), 43-60.

14. M. Gevrey, Démonstration du théorème de Picard-Bernstein par la méthode des contours successifs; prolongement analytique, Bull. Sci. Math. (2) 50 (1926), 113-128.

15. E. Giusti, Sulla regolarita parziale delle soluzioni di sistemi ellittici quasilineari di ordine arbitrario, Preprint.

16. E. Giusti and N. Miranda, Un esempio di soluzioni discontinue per un problema di minimo relativo ad un integrale regolare del calcolo delle variazioni, Boll. Un. Mat. Ital. (4) 2 (1968), 1-8.

17. A. Haar, Über das Plateausche Problem, Math. Ann. 97 (1927), 127-158.

18. D. Hilbert, Mathematische Probleme, Nachr. Acad. Wiss. Göttingen. Math.Phys. K1. Math.-Phys.-Chem. Abt. 3 (1900). 
19. —, Über das Dirichletsche Prinzip, Jber. Deutsch. Math.-Verein. 8 (1900), 184-188.

20. E. Hopf, Zum analytischen Charakter der Lösungen regulärer zwei dimensionaler Variationsprobleme, Math. Z. 30 (1929), 404-413.

21. - Über den Funktionalen, insbesondere den analytischen Charakter der Lösungen elliptischer Differentialgleichungen zweiter Ordnung, Math. Z. 34 (1932), 194-233.

22. O. A. Ladyženskaya and N. Ural'ceva, Quasilinear elliptic equations and variational problems in several independent variables, Uspehi Mat. Nauk 16 (1961), no. 1 (97) 19-90 = Russian Math. Surveys 16 (1961), 17-91.

23. - On the smoothness of weak solutions of quasi-linear equations in several variables and of variational problems, Comm. Pure Appl. Math. 14 (1961), 481-495.

24. - Linear and quasi-linear equations of elliptic type, "Nauka", Moscow, 1964; English transl., Academic Press, New York, 1968.

25. E. E. Levi, Sulle equazioni lineari totalement ellittice alle derivate parziali, Rend. Circ. Mat. Palermo 24 (1907), 275-317.

26. H. Lewy, Neuer Beweis des analytischen Charakters der Lösungen elliptischer Differentialgleichungen, Math. Ann. 101 (1929), 609-619.

27. L. Lichtenstein, Über den analytischen Charakter der Lösungen zweidimensionaler Variationsprobleme, Bull. Acad. Sci. Cracovie, Cl. Sci. Math. Nat. A, (1912), 915-941.

28. — Zur Theorie der konformen Abbildung. Konforme Abbildung nichtanalytischer singuläritätenfrei Flächenstïcke auf ebene Gebiete, Bull. Acad. Sci. Cracovie, Cl. Sci. Mat. Nat. A, (1916), 192-217.

29. E. J. McShane, Parametrizations of saddle surfaces with applications to the problem of Plateau, Trans. Amer. Math. Soc. 35 (1934), 718-733.

30. C. B. Morrey, Jr., On the solutions of quasi-linear elliptic partial differential equations, Trans. Amer. Math. Soc. 43 (1938), 126-166.

31. - Functions of several variables and absolute continuity. II, Duke Math. J. 6 (1940), 187-215.

32. - Existence and differentiability theorems for the solutions of variational problems for multiple integrals, Bull. Amer. Math. Soc. 46 (1940), 439-458.

33. - Multiple integral problems in the calculus of variations and related topics, Univ. California Publ. Math. 1 (1943), 1-130.

34. - The problem of Plateau on a Riemannian manifold, Ann. of Math. (2) 49 (1948), 807-851.

35. - On the analyticity of the solutions of non-linear elliptic systems of partial differential equations. II, Amer. J. Math. 80 (1958), 219-234.

36. - Extensions and applications of the De Giorgi-Nash results, Proc. Sympos. Pure Math., vol. IV, Amer. Math. Soc., Providence, R. I., 1961.

37. - Existence and differentiability theorems for variational problems for multiple integrals. Partial differential equations and continuum mechanics, Univ. of Wisconsin Press, Madison, 1961, pp. 241-270.

38. - Multiple integrals in the calculus of variations, Die Grundlehren der Math. Wissenschaften, Band 130, Springer-Verlag, New York, 1966.

39. - Partial regularity results for non-linear elliptic systems, J. Math. Mech. 17 (1968), 649-670.

40. C. B. Morrey, Jr. and L. Nirenberg, On the analyticity of the solutions of linear elliptic systems of partial differential equations, Comm. Pure Appl. Math. 10 (1957), 271-290. 
41. J. Moser, $A$ new proof of De Giorgi's theorem concerning the regularity problem for elliptic differential equations, Comm. Pure Appl. Math. 13 (1960), 457-468.

42. J. Nash, Continuity of the solutions of parabolic and elliptic equations, Amer. J. Math. 80 (1958), 931-954.

43. J. Neças, Les méthodes directes en théorie des équations elliptiques, Academia, Prague, 1967.

44. - Sur la régularité des solutions variationelles des équations elliptiques nonlineaires d'ordre $2 k$ en deux dimensions, Ann. Scuola Norm. Sup. Pisa 21 (1967), 427457.

45. L. Nirenberg, Remarks on strongly elliptic partial differential equations, Comm. Pure Appl. Math. 8 (1955), 648-674.

46. R. Palais and S. Smale, $A$ generalized Morse theory, Bull. Amer. Math. Soc. 70 (1964), 165-172.

47. I. PetrovskiY̌, Sur l'analyticité des solutions des systèmes d'équations différentielles, Mat. Sb. 5 (47) (1939), 3-70.

48. T. Rado, Das Hilbertsche Theorem über den analytischen Charakter der Lösungen der partiellen Differentialgleichungen zweiter Ordnung, Math. Z. 25 (1926), 514-589.

49. - Über zweidimensionale reguläre Variationsprobleme, Math. Ann. 101 (1929), 620-632.

50. - On the problem of least area and the problem of Plateau, Math. Z. 32 (1930), 763-796.

51. E. R. Reifenberg, Solution of the Plateau problem for m-dimensional surfaces of varying topological type, Acta Math. 104 (1960), 1-92.

52. - An epiperimetric inequality related to the analyticity of minimal surfaces, Ann. of Math. (2) 80 (1964), 1-14.

53. - On the analyticity of minimal surfaces, Ann. of Math. (2) 80 (1964), 15-21.

54. A. G. Sigalov, Two-dimensional problems of the calculus of variations, Uspehi Mat. Nauk 6 (1951), no. 2 (42), 16-101; English transl., Amer. Math. Soc. Transl. (1) 6 (1962), 27-146.

55. S. L. Sobolev, On a theorem of functional analysis, Mat. Sb. 4 (46) (1938), 471-497; English transl., Amer. Math. Soc. Transl. (2) 34 (1963), 39-68.

56. L. Tonelli, Sui massimi e minimi assoluti del calcolo delle variazione, Rend. Circ. Mat. Palermo 32 (1911), 297-337.

57. - Sur caso regolare nel calcolo delle variazioni, Rend. Circ. Mat. Palermo 35 (1913), 49-73.

58. - Sur une méthode directe du calcul des variations, Rend. Circ. Mat. Palermo 39 (1915), 233-264.

59. — La semicontinuita nel calcolo delle variazioni, Rend. Circ. Mat. Palermo 44 (1920), 167-249.

60. —-, Fondamenti del calcolo delle variazioni. I, II, III, Zanichelli, Bologna 61. - Sur la semi-continuité des intégrales doubles du calcul des variations, Acta Math. 53 (1929), 325-346.

62. —— L'estremo assoluto degli integrali doppi, Ann. Scuola Norm. Sup. Pisa (2) 3 (1933), 89-130.

University of California, Berkeley, California 94720 